\title{
Intraoperative Parathyroid Hormone is a Valuable Indicator of Long Term Cure in Primary Hyperparathyroidism
}

\author{
Florbela Ferreira $^{\mathrm{a}, \mathrm{e}}$, Paula Font ${ }^{\mathrm{b}}$, Deolinda Madureira ${ }^{\mathrm{b}}$, Jorge Rosa Santos ${ }^{\mathrm{c}}$, Valeriano Leite ${ }^{\mathrm{d}}$
}

\begin{abstract}
Background: The use of intraoperative parathyroid hormone (ioPTH) during surgery for primary hyperparathyroidism (PHPT) has become widely available as a tool to monitor the success of the procedure. The aim of our study was to correlate the decrease of ioPTH with long term outcome in patients with PHPT.
\end{abstract}

Methods: During a 10 year period, in 137 consecutive patients with PHPT, serum PTH was measured during surgery at baseline and 5,10 and 20 minutes after excision of the suspected parathyroid gland. Surgery was considered successful if a 50\% drop in ioPTH was observed after 10 minutes. Two groups were defined - with normal (I) and above normal (II) PTH after 10 minutes. Calcium and PTH were monitored at 1.5, 3, 6 and 12 months of follow-up.

Results: Group I had significantly lower median weight of glands (1.4 vs $3.0 \mathrm{~g}$ ), maximum pre-operative calcium (11.1 vs $11.7 \mathrm{mg}$ / $\mathrm{dL})$ and ioPTH at 10 minutes (30.1 vs $124.9 \mathrm{pg} / \mathrm{mL}$ ) than group II - P $<0.05$. Serum PTH levels at 3 and 6 months of follow-up were also significantly lower in group I than group II (55.0 vs $124.5 \mathrm{pg} /$ $\mathrm{mL}$ and $55.9 \mathrm{vs} 83.1 \mathrm{pg} / \mathrm{mL}$, respectively) $-\mathrm{P}<0.05$. At 6 months, $74.3 \%$ of the patients in group I presented normal calcium and PTH, whereas in group II normal calcium and high PTH was the predominant pattern $(59.1 \%)-\mathrm{P}=0.01$.

Conclusions: Obtaining normalization of ioPTH during surgery is

Manuscript accepted for publication August 28, 2013

${ }^{a}$ Department of Endocrinology, Diabetes and Metabolism, Santa Maria Hospital, Lisbon, Portugal

${ }^{\mathrm{b}}$ Department of Endocrine Laboratory, Francisco Gentil Portuguese Oncology Institute, Lisbon, Portugal

${ }^{c}$ Department of Head and Neck Surgery, Francisco Gentil Portuguese Oncology Institute, Lisbon, Portugal

${ }^{\mathrm{d}}$ Department of Endocrinology, Francisco Gentil Portuguese Oncology Institute, Lisbon, Portugal; CEDOC, Faculty of Medical Sciences,

New University of Lisbon, Portugal

${ }^{\mathrm{e}}$ Corresponding author: Florbela Ferreira, Department of

Endocrinology, Diabetes and Metabolism, Santa Maria

Hospital, Avenida Egas Moniz, 1649-035, Lisbon, Portugal.

Email: florbela.b.ferreira@gmail.com

doi: http://dx.doi.org/10.4021/jem189w important in addition to the classic criterion of $50 \%$ decrease from baseline to predict cure of PHPT.

Keywords: Hyperparathyroidism; Cure; Intraoperative; Parathyroid hormone

\section{Introduction}

Primary hyperparathyroidism (PHPT) results from inappropriate autonomous overproduction of parathyroid hormone (PTH) by one or more parathyroid glands, which results in hypercalcemia. It affects $0.3 \%$ of the general population and $1-3 \%$ of postmenopausal women [1]. Most cases of PHPT (over $80 \%$ ) are caused by a single enlarged adenoma. Multiglandular involvement and carcinoma are less common. Most cases are sporadic but in approximately $5 \%$ of the patients it is associated with a multiple endocrine neoplasia syndrome or other rare genetic disorders [2].

Surgery is indicated for all symptomatic patients and asymptomatic cases that fulfill one of the following criteria: age under 50 years, calcemia $1 \mathrm{mg} / \mathrm{dL}$ above the upper limit of normal, osteoporosis, glomerular filtration rate under 60 $\mathrm{mL} / \mathrm{min}[3]$.

Sonography and ${ }^{99 m} \mathrm{Tc}$-sestamibi scintigraphy are the preferred imaging techniques for preoperative location of parathyroid adenomas. The sensitivity of Sestamibi scanning for single adenomas can be as high as $90 \%$ in some series, less in cases of double adenomas or gland hyperplasia [4]. Ultrasonography is painless, noninvasive, and inexpensive and can identify coexistent thyroid pathology and intrathyroidal parathyroid adenomas [5]. Contrast-enhanced CT and MRI are usually used after failure of the previous methods [6].

PTH has a short half-life of only 3 - 5 minutes, due to high liver metabolism and kidney excretion and its decrease during surgery should be able to predict the success of the procedure [7]. Nussbaum et al [8] were the first to use rapid intraoperative PTH (ioPTH) assessment to confirm the removal of all hyperfunctioning parathyroid tissue. The use of ioPTH has been validated by others as a useful tool to moni- 


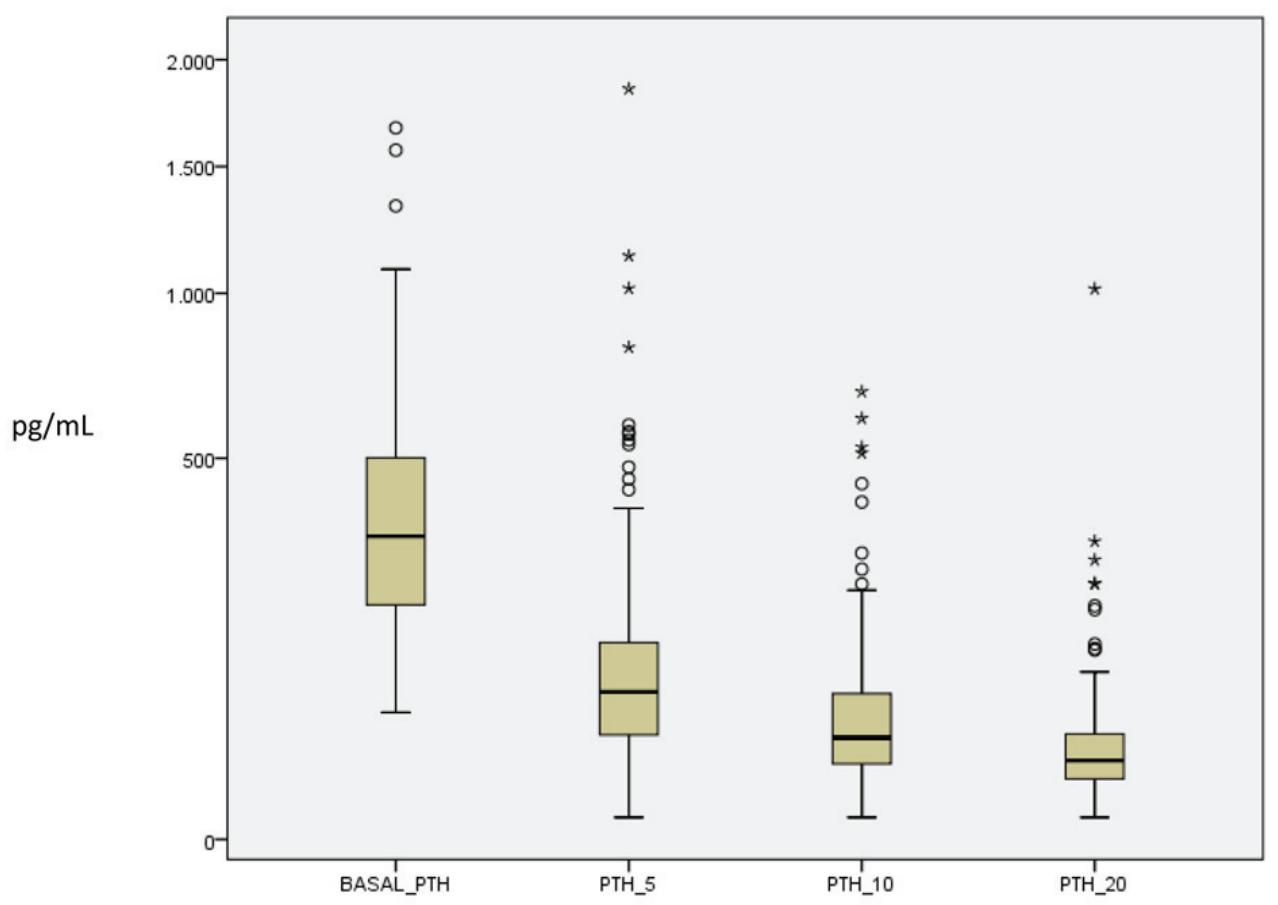

Figure 1. Boxplot chart of the PTH levels before and 5, 10 and 20 minutes after excision of the parathyroid gland.

tor the success of the procedure [4-6, 9-14].

The aim of our study was to correlate the decrease of ioPTH levels with long term outcome in patients with PHPT.

\section{Methods}

Medical records of 141 consecutive patients with primary hyperparathyroidism submitted to elective parathyroidectomy with ioPTH monitoring in Francisco Gentil Portuguese Oncology Institute were analyzed. Four patients were excluded due to scarce pre and post operative data, leaving a total of 137 patients. Surgeries were performed by one of five surgeons in the Head and Neck Department between January 2001 and July 2011 (126 months).

Patients were submitted to pre-operative analytical evaluation, including basic metabolic panel and evaluation of serum calcium, phosphate and plasma PTH. One or more imaging methods (sonography, Sestamibi scintigraphy, CT scan and/or MRI of the neck) to identify the affected gland were performed.

Patients were admitted to the inpatient department of Surgery of the Head and Neck the day before surgery. Before discharge, evaluation of calcium and phosphate were performed and patients were evaluated by an Endocrinologist. Calcium, with or without vitamin D supplements, was prescribed when calcium levels (corrected for albumin level) were below normal range. Regular follow up was then scheduled in the Endocrinology Outpatient Clinic, for clinical and laboratory evaluation, approximately at 1.5, 3, 6 and 12 months after surgery, and annually afterwards.

Intraoperative blood samples were collected from a peripheral vein to a EDTA containing tube at the time of anesthesia induction, immediately before incision, and 5, 10 and 20 minutes after excision of the affected gland. The tubes were transported to the laboratory immediately and analyzed within 5 minutes.

Intraoperative plasma intact PTH was measured using Immulite $^{\circledR} 1000$ turbo intact PTH (Siemens Healthcare), a double antibody chemiluminesce immunometric method. This assay, which requires binding by both enzyme-labeled and solid-phase antibodies, is highly specific for intact PTH. There is about $80 \%$ cross reactivity with very large aminoterminal truncated fragments (PTH 7 - 84). Reference value of PTH was $12-65 \mathrm{pg} / \mathrm{mL}$. Time for analysis of the samples was about eighteen minutes, and total time to communicate the result to the operating room was around twenty-five minutes. Reference value for calcium was $8.5-10.2 \mathrm{mg} / \mathrm{dL}$.

Measurement of PTH at follow up visits was obtained using the same method as in the intraoperative procedure.

According to the pre-operative tumor localization, when available, the surgeon directed and limited the size of the neck incision to the anatomical location identified. The incision was then closed and the patient kept under light anesthesia while awaiting the results from the laboratory. If the fall in ioPTH between the highest value before exci- 


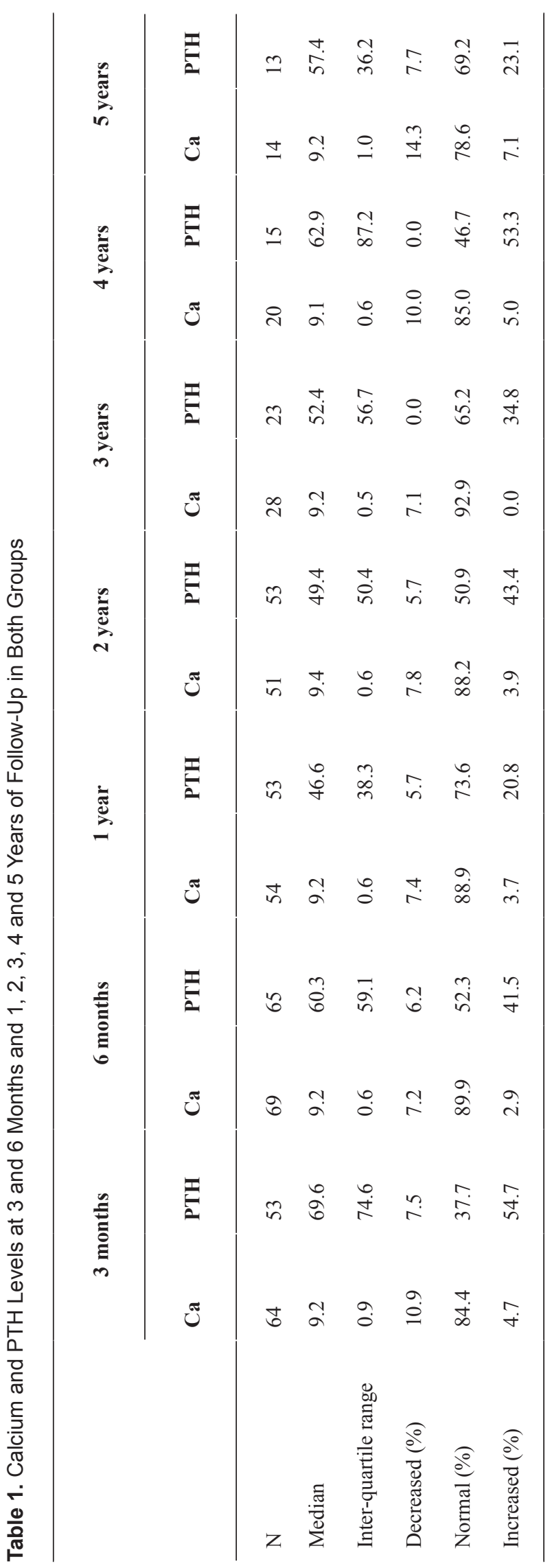

sion and the value ten minutes after excision was equal or above $50 \%$, surgery was considered successful and finished. If the decrease was inferior to the established cut-off value, the incision was reopened and additional neck exploration was performed. In all patients the excised tissue was sent for pathological examination.

In 129 cases, both baseline and 10 minutes samples were available. We defined two groups among the patients who fulfilled the 50\% PTH drop during surgery $(\mathrm{N}=125)$. Group I had PTH within the normal range and group II had an elevated level of PTH 10 minutes after surgery. We studied the differences between these two groups.

Clinical and laboratory data of the patients included in the study was collected retrospectively and included in a data base. Statistical analysis was performed using SPSS 20.0 software for Windows (SPSS Inc, Chicago, USA). Descriptive statistics were performed. Chi-square or Fisher exact test were used to compare categorical variables and the Mann-Whitney test were used to compare continuous variables between groups. To compare related samples the nonparametric Wilcoxon signed rank test was applied. Calcium and PTH levels and weight of the glands were not normally distributed and were expressed as median and interquartile range. The other continuous variables were expressed as mean and standard deviation. A P value below 0.05 was considered statistically significant.

\section{Results}

The majority of our patients were women $(78.1 \%)$, in the sixth to eighth decade of life (69.3\%). Mean age was $61 \pm$ 16 years. The median higher pre-operative levels of calcium and PTH were, respectively, $11.3 \mathrm{mg} / \mathrm{dL}$ (to convert calcium to millimoles per liter, multiply by 0.25 ) and $209.5 \mathrm{pg} / \mathrm{mL}$ (to convert to nanograms per liter, multiply by 1 ).

We considered for each patient the highest of the two basal measurements obtained, at the induction of anesthesia and pre-excision, to calculate the percentage of PTH drop during the procedure. Median level of the highest basal PTH was $323 \mathrm{pg} / \mathrm{mL}$. Most patients had measurements at 5, 10 and 20 minutes after excision. Figure 1 is a graphic representation of the drop in ioPTH during the procedure.

The mean percentage drop in PTH between the highest pre excision level and the level at 10 minutes post excision was $83.4 \pm 14.5 \%$. Almost all patients $(96.9 \%, \mathrm{n}=125)$ fulfilled the criteria of a drop equal or above $50 \%$ from baseline. PTH at 10 minutes post-excision was within the normal range in $68.0 \%$ of these patients $(\mathrm{n}=85$, median $30.1 \mathrm{mg} /$ $\mathrm{dL})$, while in the remainder of the cases was elevated $(n=40$, median $125.5 \mathrm{mg} / \mathrm{dL}$ ).

All patients had results from at least one imaging method before surgery, the average being 1.9 methods per patient. A neck sonography was performed in $81.8 \%$ cases and a ses- 
Table 2. Findings in Patients Who Fulfilled the Criterion of $50 \%$ Drop in ioPTH With Normal (Group I) or Increased (Group II) PTH After 10 Minutes Post-Excision

\begin{tabular}{|c|c|c|c|}
\hline & Group I & Group II & $\mathbf{P}$ \\
\hline Age (years) & $59.88 \pm 15.89$ & $62.93 \pm 16.14$ & 0.34 \\
\hline Gender & & & $<0.01$ \\
\hline Female (nr patients) & 74 & 24 & \\
\hline Male (nr patients) & 11 & 16 & \\
\hline Weight removed glands (g) & $1.4(2.2)$ & $3.0(6.0)$ & $<0.01$ \\
\hline Final diagnosis & & & 0.15 \\
\hline Single adenoma (nr patients) & 75 & 41 & \\
\hline Multiglandular disease (nr patients) & 11 & 2 & \\
\hline Highest pre-operative calcium (mg/dL) & $11.1(1.4)$ & $11.7(1.8)$ & $<0.01$ \\
\hline Highest pre-excision PTH & $259(210.5)$ & $863(1186.5)$ & $<0.01$ \\
\hline Intra-operative PTH - 10’ (pg/mL) & $30.1(20.5)$ & $125.5(124.9)$ & $<0.01$ \\
\hline Calcium 1.5 months (mg/dL) & $9.2(0.8)$ & $9.1(1.1)$ & 0.41 \\
\hline Calcium 3 months (mg/dL) & $9.4(0.8)$ & $9.0(1.1)$ & 0.39 \\
\hline Calcium 6 months (mg/dL) & $9.2(0.6)$ & $9.2(0.8)$ & 0.83 \\
\hline Calcium 12 months (mg/dL) & $9.2(0.6)$ & $9.2(0.7)$ & 0.59 \\
\hline PTH 1.5 months (pg/mL) & $58.0(47.2)$ & $73.4(98.7)$ & 0.16 \\
\hline PTH 3 months (pg/mL) & $55.0(49.9)$ & $124.5(136.7)$ & $<0.01$ \\
\hline PTH 6 months $(\mathrm{pg} / \mathrm{mL})$ & $55.9(48.2)$ & $83.1(92.6)$ & $<0.01$ \\
\hline PTH 12 months (pg/mL) & $46.3(39.6)$ & $61.0(57.6)$ & 0.07 \\
\hline Calcium and PTH at 6 months & & & 0.01 \\
\hline Normal calcium/normal PTH (nr patients) & 26 & 9 & \\
\hline Normal calcium/ high PTH (nr patients) & 9 & 13 & \\
\hline
\end{tabular}

Weight of the glands and levels of calcium and PTH are expressed as median and interquartile range. SI conversion factors: to convert calcium to millimoles per liter, multiply by 0.25 ; to convert PTH to nanograms per liter, multiply by 1 .

tamibi scan in $66 \%$. Neck CT scan or MRI was obtained in only $22.6 \%$ and $15.3 \%$ of the cases, respectively.

Final diagnosis was single adenoma in $88.3 \%$ of the cases, multiple adenoma in $2.2 \%$, hyperplasia in $7.3 \%$ and carcinoma in the remaining $1.5 \%$ ( 2 patients). In four cases of single adenomas, patients had had previous thyroid surgery (total or partial thyroidectomy) and localization of the culprit lesion could not be determined precisely. The remaining single adenomas were: left inferior $42.2 \%$, right inferior $42.2 \%$, left superior $11.2 \%$, right superior $4.3 \%$.

In a total of five surgeries, the wound had to be reopened during the procedure and bilateral exploration was performed due to failure of inadequate (under $50 \%$ ) fall of ioPTH 10 minutes after the removal of the suspected gland. 
They all had subsequent measurements of ioPTH. For statistical purposes we considered the values obtained in the last measurement. In two cases, the final diagnosis was parathyroid hyperplasia of three glands. In another case, the sonography identified one enlarged gland, but final diagnosis was adenoma of the contralateral parathyroid. In two cases the pre-operative imaging methods identified only one gland but two glands had to be removed to obtain a significant drop in ioPTH, and final diagnosis was of double adenoma.

Table 1 indicates the median, interquartile range and percentage of normal, decreased and elevated levels of calcium and PTH at different time points until 5 years after surgery (groups I and II). After 3 months, most patients had normal calcium (84.4\%) and this proportion increased over time (almost $90 \%$ at 6 and 12 months). The proportion of patients with normal PTH also increased over time $-37.7 \%$, $52.3 \%$ and $73.6 \%$ at 3,6 and 12 months, respectively. After the first year of follow up, nearly half of the patients had normal PTH. After 3 years $93 \%$ of the patients maintained normal levels of calcium and at 5 years after surgery $79 \%$ were normocalcemic.

We compared pre and post operative factors associated with normalization (Group I) or elevation (Group II) of ioPTH at 10 minutes, in cases where the $\geq 50 \%$ drop in ioPTH criteria was verified (Table 2). Median calcium was not statistically different between the groups at different times in follow-up, and neither was PTH at 6 weeks and one year after surgery. Having normal or high ioPTH at $10 \mathrm{~min}-$ utes post-excision was independent of the disease affecting one or multiple glands. Group I patients had significantly lower median weight of glands ( $1.4 \mathrm{vs} 3.0 \mathrm{~g}$ ), maximum preoperative calcium (11.1 vs $11.7 \mathrm{mg} / \mathrm{dL}$ ), basal ioPTH (259 vs $863 \mathrm{pg} / \mathrm{mL}$ ), and ioPTH at 10 minutes (124.9 vs $30.1 \mathrm{pg} /$ $\mathrm{mL}$ ). Serum PTH levels at 3 and 6 months of follow-up were also significantly lower in group I than in group II (55.0 vs $124.5 \mathrm{pg} / \mathrm{mL}$ and 55.9 vs $83.1 \mathrm{pg} / \mathrm{mL}$, respectively) $-\mathrm{P}<$ 0.01 .

Six month after surgery almost two thirds of the patients (59.4\%) presented normal calcium and $\mathrm{PTH}$; the remaining had elevated PTH but normal calcium. None had elevated calcium and PTH. The difference in outcomes at 6 months between groups was statistically significant $(P=0.01)$, with a higher proportion of patients presenting normal calcium and PTH in group I (26 vs 9) and with normal calcium but elevated PTH in group II (13 vs 9).

One year after surgery, $3.7 \%$ of the patients (from group II), were found to have elevated calcium and PTH, after having had normal values for the first 6 months, indicating recurrence (data not shown).

\section{Discussion}

Since it was first suggested by Nussbaum in 1988, intraop- erative measurement of PTH has been extensively used to limit the extent of surgery for PHPT with high rate of success $[9,10,12-18]$. When the classic "more than $50 \%$ drop" criterion is used, a peripheral PTH level decreasing more than $50 \%$ ten minutes after removal of the abnormal parathyroid glands predicts normal or low postoperative calcemia with an accuracy of $97-98 \%$ [19].

We used the highest pre-value of PTH (between the pre-incision and pre-excision) to calculate the percent of decrease of the ioPTH at 10 minutes, as some authors have suggested that failure to collect and compare both baseline samples increases the incidence of false negative results [20]. In our series, if we had not followed this criterion, at least some of the cases would have not fulfilled the $50 \%$ drop at 10 minutes.

Less invasive surgical procedures require pre-operative localization of the hyperfunctioning parathyroids. Ultrasonography is able to localize up to $80 \%$ of parathyroid adenomas and with Tc99m Sestamibi scanning this rate sometimes exceeds $90 \%$ [6]. The combination of multiple methods increases sensitivity in the detection of the disease. The vast majority had two preoperative imaging methods prior to surgery. The combined results of these methods allowed correct pre-operative localization of the affected gland(s) in $97 \%$ of the patients.

In five cases (4\% of the patients), the preoperative imaging methods misidentified the affected gland or failed to identify all the glands in cases of multiglandular disease. Monitoring of ioPTH was crucial in these particular cases in avoiding unnecessary reoperation. Other authors have reported a change in operative management of $8.7 \%$ after ioPTH monitoring [13].

We found a considerably increased prevalence of adenomas in the inferior parathyroids ( $84.4 \%)$. Similar results were obtained by other authors [16]. We were unable to provide logical explanation for this fact, though the results seem to indicate that this distribution is more than merely incidental.

The "hungry bone syndrome" is the fall in serum calcium and increase in influx of calcium into bone after parathyroidectomy, due to functional or relative hypoparathyroidism. It occurs in approximately $13 \%$ of the patients after this type of surgery [21]. We found a similar prevalence of hypocalcemia $(10.9 \%)$ in our patients three months after surgery. At this time point $95 \%$ had a normal or above normal level of PTH, which can be due to the hungry bone syndrome.

The proportion of patients with normal calcium is 85 $90 \%$ after surgery. An increasing percentage of patients presents with normal PTH over time - $37.7 \%, 52.3 \%$ and $73.6 \%$ at 3, 6 and 12 months, respectively. These findings emphasize the importance of regular and long term follow-up of these patients. We were able to collect data two to five years after surgery in some patients. Nearly $80 \%$ of these patients maintained normal calcemia in the long term, although the percentage of patients with a normal PTH over time is some- 
what lower $(70 \%$ at five years, probably reflecting vitamin $\mathrm{D}$ deficiency).

We were particularly interested in studying the patients who achieved a normal PTH during surgery, in addition to the classic criterion of 50\% drop in PTH. Two groups, with normal and elevated PTH after excision of the affected gland were defined, as shown in Table 2. We found that patients who had larger glands, and higher preoperative calcium and PTH were more likely to have elevated PTH at 10 minutes, even though a drop in PTH above 50\% from the highest baseline level had occurred.

Although there were no significant differences in calcium levels during the first year of follow-up, nor in PTH levels in the first 6 weeks after surgery, group II presented significantly higher levels of PTH after 3 and 6 months. At 6 months after surgery, group I had a higher proportion of patients with normal calcium and PTH (26 vs 9), whereas in group II normal calcium with elevated PTH was more prevalent ( 13 vs $9, \mathrm{P}<0.05)$. The normalization of ioPTH during surgery seems to be also associated with a lower rate of recurrence, since the two patients that recurred at 12 months were from group II.

Our results are in accordance with what has been published by other authors, indicating a higher incidence of persistent HPT in patients who did not achieve normal PTH values during surgery $[22,23]$. However, in a study conducted with a large number of patients [24] the incidence of persistent HPT was not increased in the group that did not reach normal ioPTH.

In conclusion, the majority of the patients who fulfilled the classic $50 \%$ drop criterion had normal calcium levels one year after surgery. Patients who had normal PTH after 10 minutes had lower PTH at follow-up than patients with elevated PTH at this time point. Normalization of ioPTH during surgery is associated with a smaller risk of disease persistence and a more favorable outcome.

\section{Acknowledgement}

We would like to thank all of our colleagues in the Departments of Endocrine Laboratory, Head and Neck Surgery, and Endocrinology of the Francisco Gentil Portuguese Oncology Institute, Lisbon, Portugal for their valuable contribution. The authors are also grateful to Associacao de Endocrinologia Oncologica, Portugal, for partially funding this work.

\section{References}

1. Melton LJ, 3rd. The epidemiology of primary hyperparathyroidism in North America. J Bone Miner Res. 2002;17(Suppl 2):N12-17.

2. Felger EA, Kandil E. Primary hyperparathyroidism.
Otolaryngol Clin North Am. 2010;43(2):417-432, x.

3. Bilezikian JP, Khan AA, Potts JT, Jr. Guidelines for the management of asymptomatic primary hyperparathyroidism: summary statement from the third international workshop. J Clin Endocrinol Metab. 2009;94(2):335339.

4. Civelek AC, Ozalp E, Donovan P, Udelsman R. Prospective evaluation of delayed technetium-99m sestamibi SPECT scintigraphy for preoperative localization of primary hyperparathyroidism. Surgery. 2002;131(2):149-157.

5. Udelsman R, Pasieka JL, Sturgeon C, Young JE, Clark $\mathrm{OH}$. Surgery for asymptomatic primary hyperparathyroidism: proceedings of the third international workshop. J Clin Endocrinol Metab. 2009;94(2):366-372.

6. Johnson NA, Tublin ME, Ogilvie JB. Parathyroid imaging: technique and role in the preoperative evaluation of primary hyperparathyroidism. AJR Am J Roentgenol. 2007;188(6):1706-1715.

7. Maier GW, Kreis ME, Renn W, Pereira PL, Haring HU, Becker HD. Parathyroid hormone after adenectomy for primary hyperparathyroidism. A study of peptide hormone elimination kinetics in humans. J Clin Endocrinol Metab. 1998;83(11):3852-3856.

8. Nussbaum SR, Thompson AR, Hutcheson KA, Gaz RD, Wang CA. Intraoperative measurement of parathyroid hormone in the surgical management of hyperparathyroidism. Surgery. 1988;104(6):1121-1127.

9. Sokoll LJ, Drew H, Udelsman R. Intraoperative parathyroid hormone analysis: A study of 200 consecutive cases. Clin Chem. 2000;46(10):1662-1668.

10. Dominguez JM, Velasco S, Goni I, Leon A, Gonzalez H, Claure R, Arteaga E, et al. [Usefulness of intraoperative parathormone measurement to predict surgical cure in primary hyperparathyroidism]. Rev Med Chil. 2009;137(12):1591-1596.

11. Ypsilantis E, Charfare H, Wassif WS. Intraoperative PTH Assay during Minimally Invasive Parathyroidectomy May Be Helpful in the Detection of Double Adenomas and May Minimise the Risk of Recurrent Surgery. Int J Endocrinol. 2010;2010:178671.

12. Hathaway TD, Jones G, Stechman M, Scott-Coombes D. The value of intraoperative PTH measurements in patients with mild primary hyperparathyroidism. Langenbecks Arch Surg. 2013;398(5):723-727.

13. Calo PG, Pisano G, Tatti A, Medas F, Boi F, Mariotti $\mathrm{S}$, Nicolosi A. Intraoperative parathyroid hormone assay during focused parathyroidectomy for primary hyperparathyroidism: is it really mandatory? Minerva Chir. 2012;67(4):337-342.

14. Neves MC, Ohe MN, Rosano M, Abrahao M, Cervantes O, Lazaretti-Castro M, Vieira JG, et al. A 10-year experience in intraoperative parathyroid hormone measurements for primary hyperparathyroidism: a prospective study of 91 previous unexplored patients. J Osteoporos. 
2012;2012:914214.

15. Kauffmann P, Le Bouedec G, Ptak Y, Vennat JC, Dauplat J. [Parathyroidectomy by elective route with perioperative measurement of parathyroid hormone. Report of 80 cases]. Ann Chir. 2000;125(2):149-154.

16. Ana Belen C, Carmen Ramiro P, Alicia Calero A, Raquel Latorre, Maria Diez T, Roberto Rojo B, Marivi Collado $\mathrm{S}$, et al. Tratamento del hiperparatiroidismo primario mediante abordaje minimamente invasivo. Rev Chilena de Cirurgia. 2011;63(5):473-478.

17. Ohe MN, Santos RO, Kunii IS, Abrahao M, Cervantes $\mathrm{O}$, Carvalho AB, Lazaretti-Castro $\mathrm{M}$ et al. Usefulness of intraoperative PTH measurement in primary and secondary hyperparathyroidism: experience with 109 patients. Arq Bras Endocrinol Metab. 2006;50(5):869-875.

18. Beyer TD, Solorzano CC, Starr F, Nilubol N, Prinz RA. Parathyroidectomy outcomes according to operative approach. Am J Surg. 2007;193(3):368-372; discussion 372-363.

19. Carneiro-Pla D. Contemporary and practical uses of intraoperative parathyroid hormone monitoring. Endocr Pract. 2011;17(Suppl 1):44-53.
20. Carneiro DM, Solorzano CC, Nader MC, Ramirez M, Irvin GL, 3rd. Comparison of intraoperative iPTH assay $(\mathrm{QPTH})$ criteria in guiding parathyroidectomy: which criterion is the most accurate? Surgery. 2003;134(6):973979; discussion 979-981.

21. Brasier AR, Nussbaum SR. Hungry bone syndrome: clinical and biochemical predictors of its occurrence after parathyroid surgery. Am J Med. 1988;84(4):654-660.

22. Diez M, Ratia T, Medrano MJ, Muguerza JM, San Roman MR, Medina C, Rodriguez A, et al. [Relationship between parathormone concentration during surgery and the post-operative outcome of primary hyperparathyroidism]. Cir Esp. 2011;89(6):386-391.

23. Heller KS, Blumberg SN. Relation of final intraoperative parathyroid hormone level and outcome following parathyroidectomy. Arch Otolaryngol Head Neck Surg. 2009;135(11):1103-1107.

24. Carneiro-Pla DM, Solorzano CC, Lew JI, Irvin GL, 3rd. Long-term outcome of patients with intraoperative parathyroid level remaining above the normal range during parathyroidectomy. Surgery. 2008;144(6):989-993; discussion 993-984. 